\title{
A new colour constancy algorithm based on automatic determination of gray framework parameters using neural network
}

\author{
MOHAMMAD MEHDI FAGHIH, ZEYNAB KHOSRAVINIA \\ and MOHSEN EBRAHIMI MOGHADDAM*
}

Electrical and Computer Engineering Department, Shahid Beheshti University, G.C, Tehran, Iran

e-mail:m_faghih@sbu.ac.ir; z_khosravinia@yahoo.com; m_moghadam@sbu.ac.ir

MS received 21 April 2013; revised 20 July 2013; accepted 26 July 2013

\begin{abstract}
Colour constancy is defined as the ability to estimate the actual colours of objects in an acquired image disregarding the colour of scene illuminant. Despite large variety of existing methods, no colour constancy algorithm can be considered as universal. Among the methods, the gray framework is one of the best-known and most used approaches. This framework has some parameters that should be set with appropriate values to achieve the best performance for each image. In this article, we propose a neural network-based algorithm that aims to automatically determine the best value of gray framework parameters for each image. It is a multi-level approach that estimates the optimal values for the gray framework parameters based on relevant features extracted from the input image. Experimental results on two popular colour constancy datasets show an acceptable improvement over state-of-the-art methods.
\end{abstract}

Keywords. Colour constancy; illumination estimation; neural network; generalized gray world; classification.

\section{Introduction}

Colour is a significant descriptor in computer vision and the colour of light source has a significant influence on object colours in the scene. Therefore, the image of an object, taken by the same camera but under different illumination, appears in different colours. This colour variation introduces undesirable effects in digital images and also, it may negatively affect the performance of computer vision methods for different applications (Gijsenij et al 2012). Human visual system has the natural tendency to correct the colour deviations caused by a difference in illumination. This ability is known as colour constancy.

There are many algorithms for colour constancy (Agarwal et al 2006) which can generally be divided into three classes: first class includes approaches using low level image features

${ }^{*}$ For correspondence 
for illumination estimation. Examples of algorithms in this class are Gray-World (Buchsbaum 1980), White-Patch (Land 1997), Shades of Gray (Trezzi \& Finlayson 2004), Gray-Edge (van de Weijer et al 2007). The second class involves algorithms that use a training phase for scene illuminant estimation. The gamut mapping methods (Finlayson \& Hordley 2006; Gijsenij et al 2010), genetic based method (Ebner 2006) and neural network based algorithms (Stanikunas et al 2004; Agarwal et al 2007; Faghih \& Ebrahimi Moghaddam 2011; Akhavan \& Ebrahimi Moghaddam 2010) are samples of this class. The Last class includes algorithms that estimate the colour of light source by combining other methods or selecting best method for an image. Recent researches in colour constancy such as NIS (Gijsenij \& Gevers 2011) and CAS (Bianco et al 2010) belong to this class.

The proposed method in this paper can be categorized in the third class. It selects the best algorithm for the given image among the algorithms of Gray framework by estimating the best values for its parameters using multiple neural networks.

The rest of the paper is organized as follows: the formal definition of colour constancy and some existing colour constancy algorithms are discussed in section 2. Section 3 describes the proposed method. In section 4 , the performance of the proposed method has been evaluated using two datasets and finally, conclusion was provided in section 5.

\section{Colour constancy}

Assuming Lambertian reflectance, An image can be seen as function $f$ that depends on the colour of the light source $I(\lambda)$, the surface spectral reflectance $S(\lambda)$, and the camera sensitivity function $\rho(\lambda)$. Using this notation, the sensor responses at pixel with coordinate $(x, y)$ can be shown as follows (Gijsenij \& Gevers 2011):

$$
\mathrm{f}(x, y)=\int_{\omega} I(\lambda) s(x, y, \lambda) \rho(\lambda) d \lambda,
$$

where $\omega$ is the visible spectrum, it is assumed that the scene is illuminated by one light source and that the observed colour of the light source $\overrightarrow{\mathbf{e}}$ depends on the colour of the light source $I(\lambda)$ as well as the camera sensitivity function $\rho(\lambda)$ :

$$
\overrightarrow{\mathbf{e}}=\left(\begin{array}{l}
\boldsymbol{e}_{R} \\
\boldsymbol{e}_{G} \\
\boldsymbol{e}_{B}
\end{array}\right)^{T}=\int_{\omega} I(\lambda) \rho(\lambda) d \lambda
$$

Given the image values, colour constancy can be achieved by estimating the colour of the light source $\overrightarrow{\mathbf{e}}$ followed by a transformation of the original image values using this illuminant estimate to produce a new image of the scene as if it were taken under a perfect white light (i.e. $(1 / \sqrt{3}, 1 / \sqrt{3}, 1 / \sqrt{3}))$. Since both $I(\lambda)$ and $\rho(\lambda)$ are unknown, the estimation of $\overrightarrow{\mathbf{e}}$ is an under constrained problem that cannot be solved without further assumptions (Ebner 2007).

For example, a most well-known assumption of colour constancy is Gray-world assumption: 'the average reflectance in a scene is achromatic' (Buchsbaum 1980). It directly follows from this assumption that any deviation from achromaticity in the average scene colour is caused 
by the scene illuminant. This implies that the colour of the light source $\overrightarrow{\mathbf{e}}$ can be estimated by computing the average colour in the image:

$$
\frac{\int s(\lambda, x) d x}{\int d x}=k . \overrightarrow{\mathbf{e}} .
$$

The integral in Eq. (3) is over the domain of the scene, the constant $k$ is between 0 for no reflectance (black) and 1 for total reflectance (white) of the incident light and is usually chosen such that the illuminant colour $\overrightarrow{\mathbf{e}}$, has unit length (van de Weijer et al 2007).

White-Patch is another well-known assumption: 'the maximum response in the RGB-channels is caused by a perfect reflectance' (Land 1997). A surface with perfect reflectance properties will reflect the full range of light that it captures. Consequently, the colour of this perfect reflectance is exactly the colour of the light source (Eq. (4)). In practice, the assumption of perfect reflectance is alleviated by considering the colour channels separately, resulting in the max-RGB algorithm. This method estimates the illuminant by computing the maximum response in the separate colour channels (Ebner 2007):

$$
\mathrm{f}(x, y)=\int_{\omega} e(\lambda) \times 1 \times \rho(\lambda) d \lambda=\overrightarrow{\mathbf{e}} .
$$

In Trezzi \& Finlayson (2004) the White-Patch and the Grey-World algorithms are shown to be special instantiations of a generalized colour constancy algorithm based on Minkowski norm. This generalized algorithm called 'shades of gray' and it can be computed by Eq. (5):

$$
\left(\frac{\int(\mathrm{f}(x))^{p} d x}{\int d x}\right)^{\frac{1}{p}}=k \cdot \overrightarrow{\mathbf{e}}
$$

For $\mathrm{p}=1$, the Eq. (5) is equal to the Grey-World assumption and for $\mathrm{p}=\infty$, it is equal to colour constancy by max-RGB.

Another well-known approach in colour constancy is based on Gray-Edge hypothesis that incorporates the higher order image statistics (in the form of image derivatives): 'the average of the reflectance differences in a scene is achromatic' (van de Weijer et al 2007):

$$
\frac{\int\left|s_{x}^{\sigma}(\lambda, x)\right| d x}{\int d x}=\vec{k} .
$$

Similar to the Grey-World based colour constancy, the Grey-Edge hypothesis can also be adapted to incorporate the Minkowski norm:

$$
\left(\frac{\int\left|\mathrm{f}_{x}^{\sigma}(x)\right|^{p} d x}{\int d x}\right)^{1 / p}=k
$$

Colour constancy based on this equation assumes that the ' $p^{t h}$ Minkowski norm of the derivative of the reflectance in a scene is achromatic' (van de Weijer et al 2007). van de Weijer et al (2007) also proposed a more general framework based on Minkowski norm that encompasses all the mentioned methods:

$$
\left(\int\left|\frac{\partial^{n} \mathrm{f}^{\sigma}(x)}{\partial x^{n}}\right|^{p} d x\right)^{1 / p}=k \cdot \overrightarrow{\mathbf{e}}^{n, p, \sigma},
$$


where $n$ is the order of the derivative, $p$ is the Minkowski-norm and derivatives are defined as the convolution of the image with the derivative of a Gaussian filter with scale parameter $\sigma$ (van de Weijer et al 2007).

This method (we call it the Gray framework) supposes that: ' $p^{\text {th }}$ Minkowski norm of nth order derivative of the reflectance in a scene is achromatic' and by changing its parameters, it is possible to generate many different colour constancy algorithms. For example:

1) $\vec{e}^{0,1,0}$ is equal to Gray-World algorithm.

2) $\overrightarrow{\boldsymbol{e}}^{0, \infty, 0}$ is equivalent to White-Patch algorithm.

3) $\overrightarrow{\boldsymbol{e}}^{0, p, 0}$ is the Shades of Gray algorithm.

4) $\overrightarrow{\boldsymbol{e}}^{0, p, \sigma}$ is the family of zero order colour constancy algorithms.

5) $\vec{e}^{1, p, \sigma}$ is the family of first order colour constancy algorithms.

6) $\overrightarrow{\boldsymbol{e}}^{2, p, \sigma}$ is the family of second order colour constancy algorithms.

The NIS (Gijsenij \& Gevers 2011) algorithm is a colour constancy algorithm based on selection and combination of basic colour constancy algorithms. In NIS method, natural image statistics are used to identify the most important characteristics of colour images. Then, based on these image characteristics, the proper colour constancy algorithm (or best combination of algorithms) is selected for a specific image. To capture the image characteristics, the Weibull parameterization is used (Gijsenij \& Gevers 2011) and using these image characteristics, the algorithm trains a classifier then, the trained classifier is used to select the best algorithm for new images.

\section{Proposed framework}

The main goal of the proposed method is to estimate the optimal values for the parameters of Gray framework. As mentioned in previous section, the Gray framework has three parameters: $n, p$ and $\sigma$ that are respectively the order of the derivative, the Mikowski norm and the scale parameter of the Gaussian derivative. The parameter $n$ determines the order of the algorithm that is usually chosen to be an integer in the range [0,2]. A zero order algorithm $(n=0)$ estimates the colour of light source considering only the pixel values of the image and does not use any information from the derivative of the image. On the other hand, the higher order algorithms $(n=1,2)$ use only the edge information obtained from the image derivative and therefore, they generally perform better colour constancy on images with more edges while comparing to the zero order algorithms.

For each zero order algorithm, the Minkowski norm $p$ determines the significance of the maximum value in each colour channel in estimation of the colour of light source; i.e., by setting the value of $p$ to infinity, the algorithm changes to the max-RGB algorithm and estimates the colour of light source considering only the maximum value in each channel; also, setting the value of $p$ to one results in the gray world algorithm that weights all image values equally to estimate the colour of light source. The role of the parameter $p$ for higher order algorithms is also similar to the zero order algorithms: it determines the significance of the maximum value of the derivative of three colour channels in estimating the colour of light source. The scale parameter $\sigma$ determines the amount of smoothing applied by the Gray framework algorithms. In general, for a small scale, the algorithm estimates the colour of light source while considering fine details from the image and therefore, it tends to be more sensitive to noise. On the other hand, a Gray algorithm with larger scales only uses coarse details of image (Basu 2002). 
The proposed method uses a training phase to estimate the optimum values of $n$ and $p$ in the Gray framework for each image. In the training phase, some image features are extracted and the proposed method learns the relationship between these features and the optimum values of $n$ and $p$ for a set of training images by means of a classifier and two neural networks. The trained classifier and neural networks are then used to estimate the best values of $n$ and $p$ for any given image based on the same image features. Although it is possible to estimate the optimum value of $\sigma$ using the same approach, this parameter in the proposed method is set to a constant value. This is because that our experiments showed that among the parameters of the Gray framework, the changes in the value of $\sigma$ has less effect on the performance of algorithms of Gray framework. The best value of parameters $n$ and $p$ for each image depends on different image features. Therefore, the proposed approach uses different feature vectors in determination of the values of $n$ and $p$.

\subsection{Image features}

Digital images convey information at different levels and in order to represent most of the image information, it is necessary to use different features at the same time. Therefore, the proposed approach extracts many features from images to represent the colour and texture. These features are: co-occurrence matrices, colour histogram, entropy, mean and standard deviation of wavelet transform, colour moments and Weibull parameters. Among these features, some of them are for representing the colour information and the others are for texture information. Our experiments showed that the optimum value of the order of derivative (parameter $n$ ) highly depends on both texture and colour information. Therefore, the feature vector that is used for estimating the best value of $n$ contains both colour and texture information. On the other hand, the texture information has less influence on the best value of the parameter $p$ and therefore, most texture relative features have been discarded from the feature vector for estimation of $p$. The next sections explain the mentioned features in more details and also tables 1 and 2 show the summary of described features for estimation of $p$ and $n$, respectively.

3.1a Image features for parameter $n$ : Gray Level 'Co-occurrence matrices' (GLCM) are frequently used statistical texture features in classification of images. These matrices represent the relative distances and orientation of pixels with co-occurring values matric (Haralick et al 1973).

The GLCM matrices characterize the texture of an image by calculating how often pairs of pixel with specific gray level values and in a specified spatial relationship occur in an image.

Table 1. Summary of described features for estimation of $n$.

\begin{tabular}{ll}
\hline Feature vector & No. of elements \\
\hline Co-occurrence matrices & 12 \\
Colour histogram & 24 \\
Entropy & 3 \\
Wavelet statistics & 20 \\
Colour moments & 6 \\
Weibull parameters & 60 \\
Total number of elements & 125 \\
\hline
\end{tabular}


Table 2. Summary of described features for estimation of $p$.

\begin{tabular}{ll}
\hline Feature vector & No. of elements \\
\hline Colour histogram & 48 \\
Colour moments & 6 \\
Weibull parameters & 60 \\
Total number of elements & 114 \\
\hline
\end{tabular}

The gray level co-occurrence matrix $P(i, j)$ is defined by first specifying a distance vector $d=$ $(\Delta x, \Delta y)$ and then counting all pairs of pixels separated by $d$ having gray levels $\mathrm{i}$ and $\mathrm{j}$. for given image I with $\mathrm{n}^{*} \mathrm{~m}$ pixels, $P$ can be defined as

$$
P(i, j)=\sum_{x=1}^{n} \sum_{y=1}^{m}\left\{\begin{array}{l}
1 \text { if } I(x, y)=i \quad \text { and } \quad I(x+\Delta x, y+\Delta y)=i \\
0
\end{array}\right\} .
$$

Then the features of contrast, correlation and energy of image can be extracted form GLCM as given below (Akilandeswari et al 2012):

$$
\begin{gathered}
\text { Contrast }=\sum_{i=0}^{G-1} \sum_{j=0}^{G-1} P(i, j)(i-j)^{2}, \\
\text { Correlation }=\sum_{i=0}^{G-1} \sum_{j=0}^{G-1} P(i, j) \times(i \times j)-\left(\mu_{x} \times \mu_{y}\right) /\left(\sigma_{x} \times \sigma_{y}\right), \\
\text { Energy }=\sum_{i=0}^{G-1} \sum_{j=0}^{G-1} P(i, j)^{2} .
\end{gathered}
$$

Since the texture features of an image contains valuable information about the edges of image, the proposed method uses this feature as part of the feature vector for the SVM classifier. In the proposed method, to compute GLCM, four distance vectors $(\mathrm{d})$ are used $([0,1],[-1,1],[-1,0]$ and $[-1,-1])$ that are defined as one neighbouring pixel in the possible four directions $\left(0^{\circ}, 45^{\circ}\right.$, $90^{\circ}, 135^{\circ}$ ) and using them to compute contrast, correlation and energy by Eqs. (9), (10) and (11). Therefore this feature is composed of 12 values (tree values for each of the four distance vectors).

'Colour histogram' represents the colour distribution of the image and is one of the most widely used image descriptors. It possesses several useful properties such as compactness, invariance and robustness with respect to the geometric transformation of the original image like rotation and scale (Bianco et al 2010).

Since the complete colour histogram is a very large feature and is also sparse in many times, in proposed method, the RGB colour space is quantized by uniformly dividing each colour axis into 8 intervals. Therefore, these features are composed of 24 ( 8 bins for each of R, G and B colour channels). Our experiments showed that the optimum value of parameter $n$ is more dependent on the texture features of the image than the colour features and therefore, the 8 bins quantization is used in estimating the value of this parameter.

'Entropy' is a statistical measure of randomness that can be used to characterize the texture of an image. It takes low values for smooth images and for images with strong edges its value 
is high (Petrov et al 2012). This feature is composed of 3 values (one value for each colour channel) and it can be calculated for each image according to Eq. (12):

$$
E=-\sum_{i=1}^{G} d(i) \log _{2} . d(i),
$$

where $G$ is the number of gray levels in the histogram of image (ranging from 0 to 255 for a typical 8-bit image) and $d(i)$ is the normalized value of each histogram bin (Petrov et al 2012).

Information about the textures and structures within the image can also be obtained using 'Wavelet decomposition'. The 2D wavelet transform uses a family of wavelet functions and their associated scaling functions to decompose the original image into different sub bands, namely, the low-low, low-high, high-low, and high-high (A, V, H, D, respectively.) sub bands (Gupta et al 2007). The proposed method also uses the wavelet decomposition in representation of the texture statistics of images. The wavelet part of the feature vector in the proposed method has 20 elements composed of the mean and variance of the three-level wavelet decomposition.

The 'colour distribution' of an image can be considered as a probability distribution and as any other probability distribution, it can be characterized uniquely by its central moments (Bianco et al 2010). Therefore, the first two central moments (mean and standard deviation) of colour distribution can be a guidance to select suitable algorithm for a given image. Consequently, the proposed method uses the first two central moments of the R, G and B colour channels of images as part of their feature vector (a vector with six values).

Image structures are valuable identification cues in determining which type of scene the image is taken from. Researches showed that the power spectrum (distribution of edge responses) of an image is characteristic for the type of scene and this distribution of edge responses can be modelled by a 'Weibull distribution' (Gijsenij \& Gevers 2011):

$$
w(x)=C \exp \left(-\frac{1}{\gamma}\left|\frac{x}{\beta}\right|\right)^{\gamma},
$$

where $x$ is the edge responses in a single colour channel, $C$ is normalization constant, $\beta>0$ is the scale parameter of the distribution and $\gamma>0$ is the shape parameter. Fitting this distribution on the edge responses of an image, the $\beta$ parameter is indicative for the image contrast (the higher value of $\beta$ indicates the more image contrast) and $\gamma$ parameter represents the grain size (the higher value of $\gamma$ represents the more fine textures) (Gijsenij \& Gevers 2011). The derivatives in the proposed method are defined as the convolution of image with the derivative of a Gaussian filter with scale parameter $\sigma$ (Freeman \& Adelson 1991):

$$
\frac{\partial^{s+t} f_{c, \sigma}}{\partial x^{s} \partial y^{t}}=f_{c} * \frac{\partial^{s+t} G_{\sigma}}{\partial x^{s} \partial y^{t}},
$$

where $*$ denotes the convolution and $s+t$ is equal to the order of the derivative.

The Weibull parameters must be computed for each channel separately. But, RGB channels are highly correlated (Gijsenij \& Gevers 2011); so, images must be converted to a decorrelated colour space before computing Weibull parameters. In this paper, the decorrelated opponent colour space is used:

$$
O_{1}=\frac{R-G}{\sqrt{2}}
$$




$$
\begin{gathered}
O_{2}=\frac{R+G-2 B}{\sqrt{6}}, \\
O_{3}=\frac{R+G+B}{\sqrt{3}} .
\end{gathered}
$$

In the proposed method, the part of feature vector that corresponds to the Weibull features has 60 values; i.e., the $\beta$ and $\gamma$ for the derivative of three colour channels with ten different values for $\sigma$.

3.1b Image features for parameter $p$ : The Minkowski norm $p$ is a global averaging operation that determines the relative weights of the image values from which the final illuminant colour is estimated. A high Minkowski norm emphasizes larger image values whereas a low Minkowski norm equally distributes weights among the image values (van de Weijer et al 2007). The optimum value of this parameter for each image highly depends on the colour information of that image and it has low dependency on the texture information. Therefore, the co-occurrence matrices, entropy and wavelet statistics features have been discarded from the feature vector corresponding to estimation of the best value of this parameter. Hence, the feature vector for estimation of $p$ only contains the colour histogram, colour moments and Weibull parameters. The computation of these features is the same as explained in the previous section except that RGB colour space in colour histogram is quantized by uniformly dividing each colour axis into 16 intervals instead of 8 intervals in the feature vector for estimation of $n$. The aim of this change in quantization intervals is to preserve more colour information as this parameter has more dependency on the colour information comparing to parameter $n$.

\subsection{Step-by-step of the proposed method}

The general structure of the proposed method is shown in figure 1. As it is shown, the proposed method has two levels. In the first level, it uses a multi class SVM classifier for determination of the best value of $n$ for each image. In the second level, it uses two MLP neural networks for estimation of the best value of $p$. One of the MLP networks is for estimation of $p$ when the SVM classifier determined the value of $n$ to be zero and another one is for the cases that the value of $n$ is determined to be one or two. The use of two MLPs is because that if we use only one MLP for all values of $n$, the network does not converge with good generalization performance. In summary, the training phase of the proposed algorithm consists of the following steps (the first four steps are for determining the optimum values of $n$ and $p$ for training images):

(i) Choose $n_{0}+n_{1}+n_{2}=n_{\text {Alg }}$ Gray algorithms ( $n_{0}$ zero order, $n_{1}$ first order and $n_{2}$ second order; $n_{0}=n_{1}=n_{2} ; n_{\text {Alg }}$ must be as large as possible).

(ii) For each training image $i m g_{i}, i \in[1, N]$ and Gray algorithm $a l g_{j}, j \in\left[1, n_{\text {alg }}\right]$, determine the performance $\epsilon_{i, j}$ (angular error) of $\operatorname{alg}_{j}$ on $i m g_{i} . N$ is the total number of training images.

(iii) Form the label vector $L \in \mathbb{R}^{1 \times N}$ with each element $L_{i}$ equal to the order of the algorithm with the highest performance on $i m g_{i}$ as follow:

$$
L_{i}=\operatorname{order}\left(\arg \min _{a l g}\left\{\epsilon_{i, j}\right\}\right) .
$$




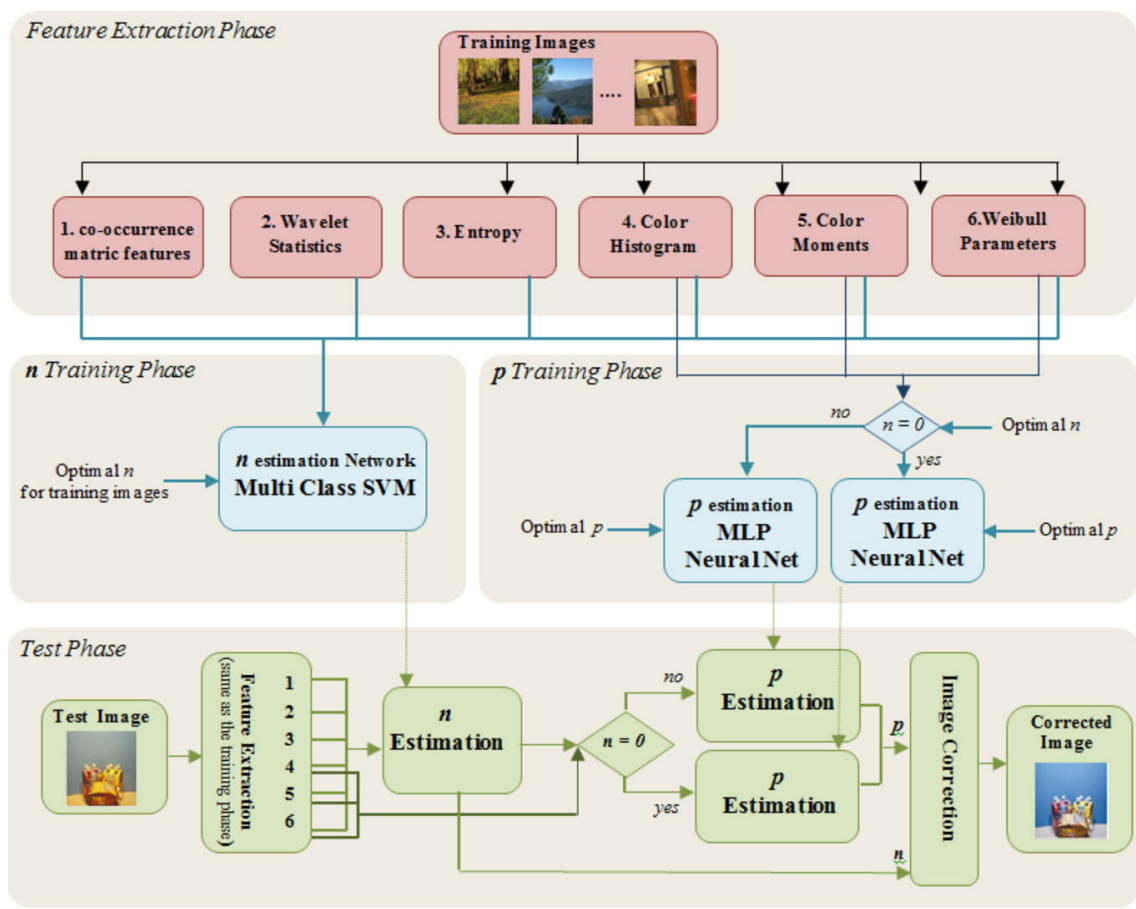

Figure 1. Overview of the proposed algorithm. The training phase consists of learning one SVM and two MLPs using the feature vectors of the training images. The illumination estimation for a given image is done by extracting the same image features and applying $t$ the Gray algorithm with the estimated parameter using the trained SVM and MLPs.

(iv) Form the Minkowski norm vector $M \in \mathbb{R}^{1 \times N}$ with each element $M_{i}$ equal to the Minkowski norm of the algorithm with the highest performance on $\mathrm{img}_{i}$ as follows:

$$
M_{i}=\text { minkowski_norm }\left(\arg \min _{\text {alg }}\left\{\epsilon_{i, j}\right\}\right) \text {. }
$$

(v) Train the SVM classifier $C$ using the following steps:

a. Extract the feature vector $f_{i}^{H} \in \mathbb{R}^{H \times 1}$ for each image $i m g_{i}$ using the process defined in section 3.1a. Build the matrix $F^{H} \in \mathbb{R}^{H \times N}$ such that each column is equal to $f_{i}^{H} . H$ is the length of the feature vector for the SVM classifier.

b. Train the classifier $C$ with the matrix $F^{H}$ and the vector $L$ as the inputs and labels respectively.

(vi) Split the training set into two subsets $S_{0}$ and $S_{1}$ based on labels of images (place the images with the label 0 into the $S_{0}$ and images with label 1 or 2 into $S_{1}$ ).

(vii) For each subset $S_{k}, k=0,1$, train the neural network $N N_{k}$ using the following steps.

a. Extract the feature vector $f_{i}^{P} \in \mathbb{R}^{P \times 1}$ for each image $i m g_{i}$ in $S_{k}$ using the process defined in section $3.1 \mathrm{~b}$. Build the matrix $F_{k}^{P} \in \mathbb{R}^{P \times N_{k}}$ such that each column is equal to $f_{i}^{P}$. 
b. For images in $S_{k}$, Form the Minkowski norm vector $M_{k} \in \mathbb{R}^{1 \times N}$ with each element $M_{i}$ equal to the Minkowski norm of the algorithm with the highest performance on img $_{i}$ as

$$
M_{i}=\text { minkowski_norm }\left(\underset{a l_{j}}{\arg \min _{j}}\left\{\epsilon_{i, j}\right\}\right) .
$$

c. Train the network $N N_{k}$ using the matrix $F_{k}^{P}$ and the matrix $M_{k}$ as the training inputs and targets, respectively.

After training phase, the normalized scene illuminant for a given image can be estimated using the following steps.

(i) Extract the feature vector $f^{H} \in \mathbb{R}^{H \times 1}$ for the given image using the process defined in section 3.1a.

(ii) Extract the feature vector $f^{P} \in \mathbb{R}^{P \times 1}$ for the given image using the process defined in section 3.1b.

(iii) Feed the $f^{H}$ to the trained classifier to determine the order of Gray algorithm for the image (parameter $n$ ).

(iv) Based on the obtained value of $n$ in previous step, feed the $f^{H}$ to the corresponding neural network to obtain the value of $p$ for the given image.

(v) Apply the Gray algorithm with the obtained values for $n$ and $p$ on the given image to estimate the scene illuminant.

\section{Experimental results}

In previous section, a new method for automatic estimation of the best values of the parameters of the Gray framework for each image has been proposed. In this section, the performance of the proposed method has been evaluated and compared with the state-of-the-art colour constancy algorithms using two public colour constancy dataset.

\subsection{Datasets}

The performance of the proposed approach has been evaluated using two public colour constancy datasets containing real world images. The first dataset was from Ciurea \& Funt (2003) dataset, a large dataset of over 11000 images. The images in this dataset have been extracted from 15 video clips taken at different locations. The real scene illuminant for each image in dataset is acquired using the small gray sphere at the bottom right corner of the images. Some example images of this dataset are shown in figure 2a. Note that while evaluating proposed approach, the gray sphere is omitted from images to avoid biasing the algorithms.

Although Ciurea and Funt dataset is a popular dataset for colour constancy, it has some disadvantages. The main disadvantage of this dataset is that the images are extracted from video sequences and therefore some of the images are correlated. Another drawback of this dataset is that the quality of images is low and also the images are processed by an unknown postprocessing procedure including gamma mapping and lossy compression. Therefore, as second dataset, the proposed approach has been tested using the Colour-Checker dataset (Lilong \& Brian 2010). The dataset consists of 568 indoor and outdoor images. The real scene illuminant for each image in this dataset is obtained using a MacBeth Colour Checker that is placed in the scene. Although this dataset contains limited number of images comparing to dataset of 

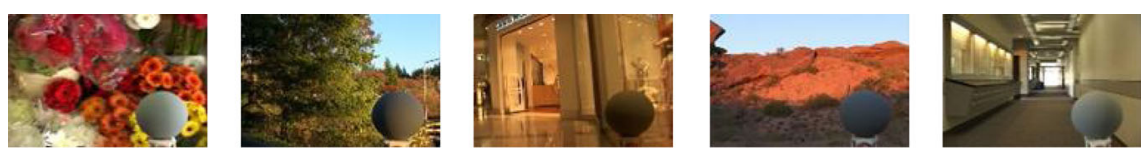

(a) some example images of Ciurea and Funt Gray-Ball dataset
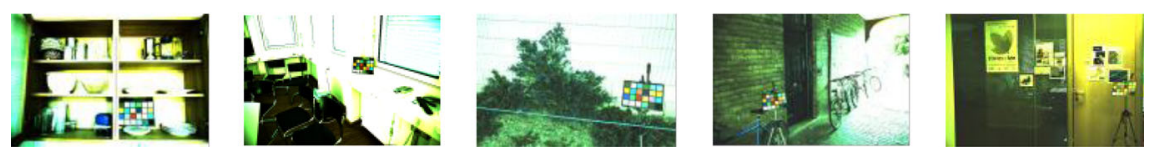

(b) some example images of Shi and Funt Color-Checker dataset

Figure 2. Example images of two popular datasets. (a) Gray-Ball dataset, (b) Colour-Checker dataset.

Ciurea \& Funt (2003), the quality of images in this dataset is high and also images are not correlated. The images in this dataset are 12 bits linear images and in the experiments, the proposed method is tested on these linear images. Also, while evaluating algorithms, the colour checker is omitted from images to avoid biasing the algorithms. Some example images of this dataset are shown in figure $2 \mathrm{~b}$. Note that a gamma correction with gamma value 2.2 is applied on these images in order to display them correctly.

\subsection{Performance measure}

One of frequently used performance measures in colour constancy research is angular error: angular distance between the estimated illuminant $\mathrm{e}_{e}$ and the ground truth $\mathrm{e}_{u}$ and is defined as Eq. (18) (Gijsenij et al 2009):

$$
d_{\text {angle }}\left(\mathrm{e}_{e}, \mathrm{e}_{u}\right)=\cos ^{-1}\left(\frac{\mathrm{e}_{\mathrm{e}} \cdot \mathrm{e}_{\mathrm{u}}}{\left\|\mathrm{e}_{e}\right\| \cdot\left\|\mathrm{e}_{u}\right\|}\right),
$$

where $\mathrm{e}_{e} \cdot \mathrm{e}_{u}$ is the dot product of the two illuminants and $\|\cdot\|$ is the Euclidean norm of a vector.

When evaluating the performance of colour constancy algorithms on a whole data set instead of on a single image, the performances on all individual images need to be summarized into a single statistic. To this end, the median angular error has been employed to measure the overall performance of algorithm on the dataset because this measure is known as the most appropriate measure for this aim (Gijsenij et al 2009). However, the error measures are often not normally distributed, but rather skewed resulting in a non-symmetric distribution. Hence, the mean value of the errors is a poor summary statistic and the most appropriate measure for this aim is the median (Gijsenij \& Gevers 2011).

Although the median angular error is an appropriate measure for comparing the overall performance of algorithms on a dataset, it does not tell us everything about the distribution of errors. Therefore, it would be better to somehow compare statistical significance of the whole error distribution of algorithms. To this end, the Wilcoxon Sign Test (Tanis \& Hogg 2001) has been used. Given two samples of random variables $A$ and $B$ the Wilcoxon sign test is used to test the null hypothesis: $H_{0}: p=P(A>B)=0.5$ which means the probability that $A$ has a value larger than $B$ in $50 \%$ of the time. In the case of colour constancy algorithms, the random variables $A$ and $B$ are the angular error results for two different algorithms and the test can be used to determine whether the performance of the algorithms are the same (the null hypothesis is true) or whether one algorithm performs significantly better than another (the null hypothesis is rejected). The 
Table 3. The performance of various colour constancy algorithms on SFU Gray-Ball dataset.

\begin{tabular}{|c|c|c|c|c|c|c|c|c|c|c|c|c|}
\hline & & $\begin{array}{l}\text { Median } \\
\text { error }\end{array}$ & $\begin{array}{l}\text { Mean } \\
\text { error }\end{array}$ & $\begin{array}{l}\text { Max. } \\
\text { error }\end{array}$ & 1 & 2 & 3 & 4 & 5 & 6 & 7 & 8 \\
\hline 1 & White patch & $5.3^{\circ}$ & $6.8^{\circ}$ & $38.7^{\circ}$ & & + & & - & - & + & - & - \\
\hline 2 & Gray world & $7.0^{\circ}$ & $7.9^{\circ}$ & $48.1^{\circ}$ & - & & - & - & - & - & - & - \\
\hline 3 & Shade of Gray $(P=12)$ & $5.3^{\circ}$ & $6.1^{\circ}$ & $41.2^{\circ}$ & & + & & - & & + & - & - \\
\hline 4 & $\begin{array}{l}\text { 1st Order Gray edge } \\
(\mathrm{P}=1, \sigma=1)\end{array}$ & $4.7^{\circ}$ & $5.9^{\circ}$ & $41.2^{\circ}$ & + & + & + & & & + & - & - \\
\hline 5 & $\begin{array}{l}\text { 2nd Order Gray edge } \\
(\mathrm{P}=1, \sigma=2)\end{array}$ & $4.9^{\circ}$ & $6.1^{\circ}$ & $41.7^{\circ}$ & + & + & & & & + & - & - \\
\hline 6 & Gamut mapping (edge based) & $5.8^{\circ}$ & $6.8^{\circ}$ & $40.3^{\circ}$ & - & + & - & - & - & & - & - \\
\hline 7 & NIS & $3.9^{\circ}$ & $5.2^{\circ}$ & $44.5^{\circ}$ & + & + & + & + & + & + & & - \\
\hline 8 & Proposed method & $3.4^{\circ}$ & $4.5^{\circ}$ & $29.0^{\circ}$ & + & + & + & + & + & + & + & \\
\hline
\end{tabular}

decision to accept or reject the null hypothesis at a given significance level $\alpha$ is made on the number of times the random variable $A$ are greater than the corresponding values of $B$.

4.2a Ciurea and Funt Gray-Ball dataset: To evaluate the performance of the proposed method on this dataset, the k-fold cross validation has been used. In k-fold cross-validation, the image data set is partitioned into $\mathrm{k}$ parts. Of the $\mathrm{k}$ parts, a single part is retained as the validation data for testing and the remaining k-1 parts are used as the training data. The cross-validation process is then repeated $\mathrm{k}$ times, with each of the $\mathrm{k}$ parts used exactly once as the validation data. The advantage of this method over repeated random subsampling is that all observations are used for both training and validation, and each observation is used for validation exactly once. In the case of the Gray-Ball dataset, because the images have been extracted from 15 video clips, the 15fold cross validation is used while making sure that the correlated images (images of the same video clip) are grouped in the same part.

Table 3 shows the median, mean and max angular error of the proposed method and some state-of-the-art algorithms on SFU Gray-Ball data set as well as the results of the Wilcoxon Sign Test on this dataset with $95 \%$ confidence interval i.e., $\alpha=.05$ (the results of algorithms other than the proposed method have been obtained from (Results per Dataset 2013)). A plus sign $(+)$ in the $i$ th row and $j$ th column of the table means that algorithm $i$ is statistically better than

Table 4. The performance of various colour constancy algorithms on Shi and Funt Colour-Checker dataset.

\begin{tabular}{llllllllllllll}
\hline & $\begin{array}{l}\text { Median } \\
\text { error }\end{array}$ & $\begin{array}{l}\text { Mean } \\
\text { error }\end{array}$ & $\begin{array}{l}\text { Max. } \\
\text { error }\end{array}$ & 1 & 2 & 3 & 4 & 5 & 6 & 7 & 8 \\
\hline 1 & White patch & 5.7 & 7.6 & 40.6 & & + & - & - & - & - & - & - \\
2 & Gray world & 6.3 & 6.4 & 24.8 & - & & - & - & - & - & - & - \\
3 & Shade of Gray $(P=4)$ & 4.0 & 4.9 & 22.4 & + & + & & + & + & + & - & - \\
4 & 1st Order Gray edge $(\mathrm{P}=1, \sigma=6)$ & 4.5 & 5.3 & 26.4 & + & + & - & & - & + & - & - \\
5 & 2nd Order Gray edge $(\mathrm{P}=1, \sigma=1)$ & 4.4 & 5.1 & 23.9 & + & + & - & + & & + & - & - \\
6 & Gamut mapping (edge based) & 5.0 & 6.5 & 29.0 & + & + & - & - & - & & - & - \\
7 & NIS & 3.1 & 4.2 & 26.2 & + & + & + & + & + & + & & \\
8 & Proposed method & 2.8 & 5.0 & 32.5 & + & + & + & + & + & + & & \\
\hline
\end{tabular}




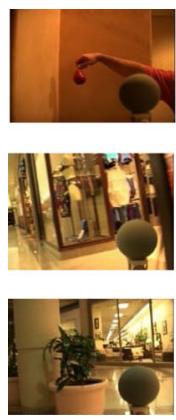

(a)
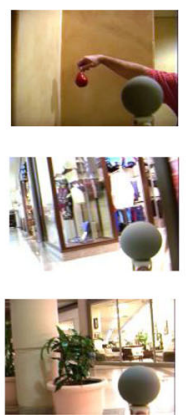

(b)

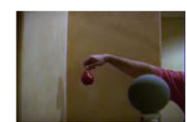

$4.2^{\circ}$

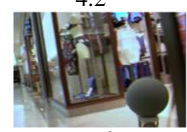

$1.0^{\circ}$

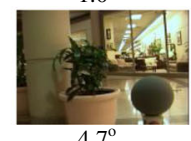

(c)

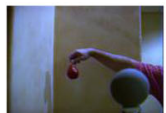

$9.5^{\circ}$

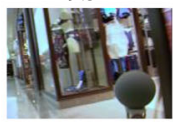

$1.6^{\mathrm{o}}$

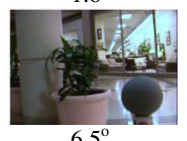

(d)

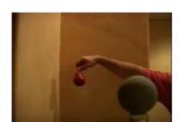

$11.1^{\circ}$

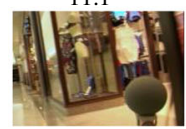

$10.2^{\circ}$

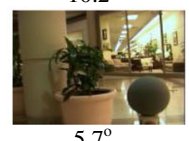

(e)

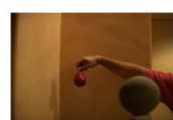

$12.4^{\circ}$

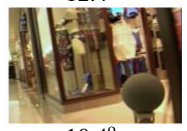

$10.4^{\circ}$

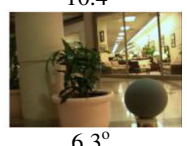

(f)

Figure 3. Examples from a real-world data set. The angular error is shown in the bottom of the images. (a) Original image, (b) ideal correction, (c) proposed method, (d) shade of Gray, (e) 1st-order Grey-Edge, (f) 2 st-order Grey-Edge.

algorithm $j$ when judged according to the Wilcoxon test. A minus (-) implies that it is worse, and if the box is empty the two algorithms are statistically the same.

On the basis of the results in table 3 , it can be concluded that on overall, the performance of the proposed method is better than other algorithms and the median, mean and max angular error and also the Wilcoxon Sign Test results support this claim. Additionally, our approach gains $12.8 \%$ and $15.5 \%$ improvement in median and mean angular error over the NIS method that is an algorithm based on the selection and combination of algorithms of the Gray framework.

4.2b Shi and Funt Colour-Checker dataset: To evaluate the performance of proposed approach on this dataset, the 3 -fold cross validation test has been used. The 3 -fold is because the author of this dataset divided the images in three categories. The median, mean and maximum angular errors as well as the results of the Wilcoxon Sign Test with 95\% confidence interval for various colour constancy algorithms on this dataset are shown in table 4 (the results of algorithms other than the proposed approach have been obtained from Results per Dataset 2013). As the table 4 shows, the proposed method gains $9.7 \%$ improvement of the median angular error over NIS method in this dataset (figure 3).

\section{Conclusion}

Despite large variety of existing colour constancy method, there is no any generalized colour constancy algorithm that can be considered as universal. This paper proposed a new automatic colour constancy selection algorithm based on estimating the optimal values of the two must effective parameters of the Gray framework using an SVM classifier and two neural networks. To achieve this goal, first, relevant features have been extracted from images and then, the extracted features have been used to train the classifier and the neural networks.

The results of experiments on two colour constancy datasets showed that our proposed method not only has better performance over the simple algorithms, but it also outperforms the NIS method that is a combining method. Our future work includes more research on feature extraction techniques and testing several classifiers to improve the efficiency of the method. 


\section{Acknowledgement}

We would like to thank Iran National Science Foundation for the financial support for this research work.

\section{References}

Agarwal V, Abidi B, Koschan A and Abidi M 2006 An overview of colour constancy algorithms. J. Pattern Recognition Research 1(1): 42-54

Agarwal V, Gribok A V and Abidi M A 2007 Machine learning approach to colour constancy. Neural Networks 20: 559-563

Akhavan T and Ebrahimi Moghaddam M 2010 A New Combining Learning Method for Color Constancy, in 2nd International Conference on Image Processing Theory, Tools and Applications (IPTA)

Akilandeswari U, Nithya R and Santhi B 2012 Review on Feature Extraction Methods in Pattern Classification. European J. Sci. Research 71(2): 265-272

Basu M 2002 Gaussian-Based Edge-Detection Methods-A Survey, IEEE Transactions on Systems, Man, and Cybernetics-Part C: Applications and Reviews 32(3): 252-260

Bianco S, Ciocca G, Cusano C and Schettini R 2010 Automatic colour constancy algorithm selection and combination. Pattern Recognition 43(3): 695-705

Buchsbaum G 1980 A spatial processor model for object colour perception. J. Franklin Institute 310(1): $1-26$

Ebner M 2006 Evolving colour constancy. Pattern Recognition Lett. 27: 1220-1229

Ebner M 2007 Colour constancy. Wiley-IS\&T Series in Imaging Science and Technology. John Wiley \& Sons

Faghih M M and Ebrahimi Moghaddam M 2011 Neural Gray Edge: Improving Gray Edge Algorithm Using Neural Network, in International Conference on Image Processing

Finlayson G D and Hordley S D 2006 Gamut Constrained Illuminant Estimation. Int. J. Comput. Vision 67: 93-109

Freeman W T and Adelson E H 1991 The Design and Use of Steerable Filters. Pattern Analysis and Machine Intelligence, IEEE Transactions on 13(9): 891-906

Funt F and Ciurea B 2003 A Large Image Database for Colour Constancy Research, in IS\&T/SID's Colour Imaging Conference. IS\&T - The Society for Imaging Science and Technology, Scottsdale

Gijsenij A and Gevers T 2011 Colour Constancy using Natural Image Statistics and Scene Semantics, IEEE Transactions On Pattern Analysis and Machine Intelligence 33(4): 687-698

Gijsenij A, Gevers T and Lucassen M P 2009 Perceptual analysis of distance measures for colour constancy algorithms. Optical Society of America 26(10): 2243-2256

Gijsenij A, Gevers T and van de Weijer J 2010 Generalized Gamut Mapping using Image Derivative Structures for Colour Constancy. Int. J. Comput. Vision 86: 127-139

Gijsenij A, Lu R and Gevers T 2012 Colour Constancy for Multiple Light Sources. IEEE Transactions on Image Processing 21(2): 697-707

Gupta L, Pathangay V and Patra A et al 2007 Indoor versus Outdoor Scene Classification Using Probabilistic Neural Network. EURASIP J. Advances in Signal Processing 2007: 1-10

Haralick R M, Shanmuga K and Dinstein I 1973 Textural features for image classification. IEEE Transactions on Systems, Man \& Cybernetics, Vols. SMC-3, 6: 610-621

Land E 1997 The Retinex Theory of Colour Vision. Scientific American 237(6): 108-128

Lilong S and Brian F 2010 Re-processed Version of the Gehler Colour Constancy Dataset of 568 Images. [Online]. Available: http://www.cs.sfu.ca/ colour/data/

Petrov N, Georgieva A and Jordanov I 2012 Self-organizing maps for texture classification. Neural Comput. and Applic.

Results per Dataset 2013 [Online]. Available: http://colorconstancy.com/?page_id=17 
Stanikunas R, Vaitkevicius H and Kulikowski J J 2004 Investigation of colour constancy with a neural network 17: 327-337

Tanis E A and Hogg R V 2001 Probability and Statistical Inference: Prentice Hall

Trezzi G D and Finlayson E 2004 Shades of Gray and Colour Constancy, in Colour Imaging Conference $I S \& T / S I D$ 's, Scottsdale, Arizona

van de Weijer J, Gevers T and Gijsenij A 2007 Edge-Based Colour Constancy. IEEE Transactions on Image Processing 16(9): 2207-2214 